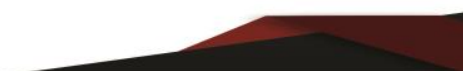

Artigo de Revisão

\title{
Qualidade de Vida e Qualidade de Vida no Trabalho: uma revisão bibliográfica
}

\author{
Roberto Medeiros da Fonsêca Cavalcante ${ }^{I}$; Karen Valverde Pontes ${ }^{2}$; Anselmo Alves Bandeira ${ }^{3}$; André Leite Rocha ${ }^{4}$
}

\begin{abstract}
Resumo: É fato que a preocupação com as tecnologias que aumentem o desempenho do trabalhador e reduza seu esforço físico é tão antiga quanto o homem moderno. Entretanto, os estudos científicos que analisam a Qualidade de Vida e a Qualidade de Vida no Trabalho são recentes, sendo que estes temas foram mais abordados nas últimas décadas. Neste sentido, este artigo apresenta uma revisão bibliográfica sobre o referencial teórico referente ao termo Qualidade de Vida (QV) e Qualidade de Vida no Trabalho (QVT), assim como seu contexto histórico e desenvolvimento como ciência.
\end{abstract}

Palavras-chave: Qualidade de Vida. Trabalho. Saúde do trabalhador.

\section{Quality of Life and Quality of Life at Work: a literature review}

\begin{abstract}
It is a fact that the concern with the technologies that increase the performance of the worker and reduces his physical effort is as old as the modern man. However, scientific studies that analyze Quality of Life and Quality of Working Life are recent, being these subjects were more approached in the last decades. In this sense, this article presents the bibliographical review about the theoretical reference referring to the term Quality of Life (QV) and Quality of Working Life (QWL), as well as its historical context and development as a science.
\end{abstract}

Keywords: Quality of Life. Work. Worker's health.

\section{Introdução}

Atualmente, as organizações estão em uma busca contínua de aperfeiçoamento e aumento da produtividade. Neste contexto, a gestão da Qualidade de Vida no Trabalho vem se mostrando decisiva para consecução dos objetivos organizacionais, pois, quando motivadas, as pessoas são capazes de trabalhar com eficácia, garantido assim o alcance das metas estabelecidas e a sobrevivência das instituições diante das adversidades do mercado.

\footnotetext{
${ }^{1}$ Mestre em Engenharia Industrial pela Universidade Federal da Bahia robertomfc@hotmail.com

${ }^{2}$ Doutora em Engenharia Química pela Universidade Estadual de Campinas e pela Universidade RWTH-Aachen na Alemanha

${ }^{3}$ Doutor em Engenharia de Produção pela Universidade de São Paulo

${ }^{4}$ Doutor em Química e Biotecnologia pela Universidade Federal de Alagoas
} 
Além da simples melhoria da produtividade dos servidores, os investimentos em QVT promovem a valorização do trabalhador com pessoa, englobando esforços voltados para saúde, bem-estar, motivação pessoal, entre outros.

Ademais, o crescimento dos estudos sobre Qualidade de Vida e Qualidade de Vida no Trabalho como ciência tem acompanhado a evolução das relações de emprego e trabalho. Partindo-se, por exemplo, dos estudos de Mayo em 1927, observou-se a influência dos fatores sociais no desempenho dos trabalhadores. Já recentemente, percebe-se que as abordagens praticadas pelas modernas organizações estão em busca do bem-estar e aumento da satisfação do trabalhador.

\section{Qualidade de Vida}

Historicamente estima-se que o termo Qualidade de Vida (QV) tenha surgido pela primeira vez na década de 30, na literatura médica, associado a trabalhos empíricos que buscavam sua definição e mensuração. No entanto, a maior acepção científica do termo se dá mais recentemente nas últimas décadas, principalmente a partir dos anos 80, quando se desenvolveram muitos instrumentos para avaliação da QV (SEIDL; ZANNON, 2004). Apesar da grande quantidade de publicações acerca da QV, segundo Landeiro et al. (2011), não existe uma definição consensual, mas os pesquisadores parecem concordar que a Qualidade de Vida é um conceito dinâmico, amplo, subjetivo e polissêmico.

Gomes et al. (2011) informam que o termo QV é bastante controverso, pois se baseia na percepção individual de um completo bem-estar físico, mental e social. A sensação de bemestar de uma pessoa deriva da satisfação ou insatisfação com as áreas da vida que são importantes para ela. A maioria dos autores concorda que em sua avaliação devem ser contemplados os domínios físico, social, psicológico e espiritual, buscando-se captar a experiência pessoal de cada indivíduo.

Diante dessa abrangência, podem-se esboçar alguns dos fins aos quais a utilização da expressão Qualidade de Vida atende: técnicos, como conceito articulador de outros conceitos ou de práticas nas ciências biomédicas; econômicos, como aglutinador de valor ou justificador da produção ou compra de produtos e serviços; clínicos, como indicador da eficácia de um procedimento de intervenção ou como medida organizadora de grupos de pacientes; político- 
administrativos, como medida de avaliação e de comparação de políticas públicas de saúde; e subjetivos, como maneira de perceber e avaliar a própria saúde ou como forma de organizar as possibilidades de seu aprimoramento (GIMENES, 2013).

Segundo Soares et al. (2011), um dos núcleos de sentido identificado foi a Qualidade de Vida como um conceito multidimensional e subjetivo. O termo Qualidade de Vida compreende diversos significados, que refletem os conhecimentos, as experiências e os valores de determinados indivíduos e sociedades, em diferentes épocas e locais, refletindo histórias de vida diferentes. Apesar das divergências citadas anteriormente, neste ponto parece haver razoável concordância entre os pesquisadores acerca do constructo QV, cujas características são: subjetividade e multidimensionalidade (MACHADO et al., 2011).

Ainda segundo Machado et al. (2011), o conceito de QV é amplo, uma vez que pode ser afetado de modo complexo por diversos fatores entre os quais pela saúde física do indivíduo, pelo seu estado psicológico, por suas relações sociais, por seu nível de independência e pelas suas relações com as características mais relevantes do meio ambiente.

Diante dos grandes avanços nas pesquisas e o crescente interesse sobre a temática, na década de 90 a Organização Mundial da Saúde reuniu especialistas sobre saúde e QV de diversas regiões do mundo para formar um grupo de estudos sobre QV, com a finalidade de aumentar o conhecimento científico da área e de desenvolver instrumentos para sua avaliação dentro de uma perspectiva transcultural (GORDIA et al., 2011).

Gordia et al. (2011) informam que em 1995 a Organização Mundial da Saúde, tentando avaliar e sintetizar todos estes fatores sobre QV, a definiu como a percepção do indivíduo sobre a sua posição na vida, no contexto da cultura e dos sistemas de valores nos quais ele vive, e em relação a seus objetivos, expectativas, padrões e preocupações. Ainda em relação à conceituação, Martin e Stockler (1998), definem a QV como a distância entre a expectativa individual e a realidade, sendo que quanto menor a distância melhor a QV.

Lipp e Rocha (1996) definem Qualidade de Vida como o viver que é bom e compensador em pelo menos quatro áreas: social, afetiva, profissional e saúde. Para que a pessoa possa ser considerada como tendo uma boa QV, torna-se necessário que ela tenha sucesso em todos esses quadrantes. Segundo os autores não adianta você ter muito sucesso só na sua carreira, ou só na área social e não o ter nas outras áreas.

Os mesmos autores defendem que por meio do processo de melhoria da QV, busca-se um estado permanente de equilíbrio entre a saúde física, o domínio, vivência das emoções e a 
elevação da consciência através de valores e crenças. A partir desse equilíbrio pessoal e interior, as relações interpessoais agregam qualidade e busca-se uma harmonia com o meio ambiente (LIPP; ROCHA, 1996).

Em um contexto atual, Barcaui e Limongi-França (2014), informam que o termo Qualidade de Vida é detentor de diversas definições, mas normalmente está associado ao bemestar dos servidores de uma empresa. Seus aspectos incluem: saúde (física e psicológica), segurança, lazer, ergonomia e o próprio ambiente de trabalho, entre outros.

\section{Qualidade de Vida no Trabalho}

A origem da Qualidade de Vida no Trabalho, de acordo com Rodrigues (1999), advém de uma preocupação antiga do homem. Os contextos eram outros, assim como as denominações utilizadas, mas sempre houve o objetivo de alcançar a satisfação do trabalhador e de reduzir o esforço físico no trabalho.

Assim, ainda segundo o autor, a satisfação no trabalho e a ênfase no desempenho estiveram sempre presentes nas relações profissionais desde os primórdios, quando métodos foram desenvolvidos e aplicados visando minimizar o mal-estar ou o esforço inadequado do trabalho. Historicamente exemplificando, Vasconcelos (2001) nos informa que os ensinamentos de Euclides (300 a.C.) de Alexandria sobre os princípios da geometria serviram de inspiração para a melhoraria do método de trabalho dos agricultores à margem do Nilo, bem como a Lei das Alavancas, de Arquimedes, formulada em 287 a.C., veio a diminuir o esforço físico de muitos trabalhadores.

Algumas premissas relacionadas às ideias de QVT também podem ser encontradas no clássico experimento conduzido por Elton Mayo em 1927 em uma fábrica da Western Electric Company, situada no bairro Hawthorne, de Chicago. Conhecido assim como "Experimento de Hawthorne", Mayo constatou que modificações nas configurações física e social do ambiente de trabalho podem provocar mudanças emocionais e comportamentais significativas nos trabalhadores, como fadiga, acidentes de trabalho, turnover e redução no desempenho e produtividade. Nesta ocasião provou-se então que os trabalhadores não estavam somente influenciados pelas condições físico-ambientais, mas também os fatores psicossociais ligados à cultura organizacional (GOMES; MENEZES, 2010). 
De acordo com Maximiano (2005), a partir dos estudos de Mayo houve o surgimento da Escola das Relações Humanas na Administração, em que se defendia que os aspectos emocionais e o comportamento coletivo dos trabalhadores nas organizações eram tão importantes quanto os métodos de trabalho. Posteriormente, na década de 40 surge a Teoria Comportamental da Administração que, segundo Chiavenato (2004), é caracterizada por ser decorrência da Teoria das Relações Humanas. Assim, sua ênfase ainda se encontra no comportamento humano, porém, leva em consideração o contexto organizacional, de forma mais ampla, abrangendo a influência desse comportamento na organização como um todo e as perspectivas das pessoas diante das organizações.

Com o enfoque comportamental, merece destaque o trabalho de Abraham H. Maslow, que concebeu a hierarquia das necessidades, composta de cinco necessidades fundamentais: fisiológicas, segurança, amor, estima e autorrealização. Douglas McGregor, autor da Teoria X, por sua vez, considerava, entre outras coisas, que o compromisso com os objetivos depende das recompensas à sua consecução, e que o ser humano não só aprende a aceitar as responsabilidades, como passa a procurá-las (VASCONCELOS, 2001). Ressalta-se que vários aspectos presentes nas obras de Maslow e McGregor ainda hoje são observados e considerados na elaboração de programas de QVT.

Há de se relatar também as importantes contribuições de Frederick Herzberg com a criação da Teoria dos Dois Fatores em 1959. Esta foi a primeira teoria proposta que explicava a satisfação com o trabalho, sugerindo dois grupos igualmente importantes para levar a satisfação da equipe: fatores higiênicos e fatores motivacionais. $\mathrm{O}$ primeiro se refere às condições físicas no trabalho, salário, benefícios, segurança, dentre outros, e os fatores que fomentam a motivação correspondem ao estado em que o trabalhador se sente com disposição ou vontade para trabalhar produtivamente (SILVEIRA; STIPP; MATTOS, 2014). Herzberg concluiu que os fatores higiênicos, quando estão ausentes, proporcionam grande insatisfação, mas sua presença não traz o mesmo nível percentual de satisfação. Portanto, para motivar um funcionário, não basta que os fatores de insatisfação estejam ausentes. Pelo contrário, os fatores de satisfação (higiênicos) devem estar bem presentes, assim como os fatores motivacionais (SILVEIRA; STIPP; MATTOS, 2014).

Entretanto, os primeiros estudos realizados com o termo propriamente dito de Qualidade de Vida no Trabalho aconteceram na década de 1950, na Inglaterra, por meio das pesquisas desenvolvidas no "Tavistock Institute" por Eric Trist e seus colaboradores. Estes pesquisadores 
desenvolveram uma abordagem sociotécnica com base em investigações sobre o trinômio indivíduo, trabalho e organização, e a análise e reestruturação da tarefa com vistas à satisfação do trabalhador (FERNANDES, 1996; VIEIRA, 1996).

Ressalta-se, porém, que o conceito Qualidade de Vida no Trabalho surge somente na década de 70 e é trabalhada por Louis Davis. Algum tempo depois da definição do conceito, esse pesquisador criou o Center for Quality of Working Life na Califórnia, Estados Unidos. Davis mantinha contato com os pesquisadores do Tavistock Institute, logo foi influenciado pelas pesquisas que eles realizaram (VELOSO; BOSQUETI; LIMONGI-FRANÇA, 2005).

Ainda na década de 70, a QVT figurou como abordagem teórica e como método, focalizando o indivíduo e seu ambiente de trabalho, utilizando-se de um conjunto de técnicas a fim de melhorar as condições laborais. Tudo isso visava tornar o trabalhador mais produtivo num contexto organizacional satisfatório. Entretanto, de modo geral, as investigações em QVT ocuparam pouco espaço na produção científica, ficando esse tema à margem das discussões acadêmicas até o final desse decênio (CAMPANA; PEÇANHA, 2010).

Nos anos 80, a abordagem da QVT passa a ser caracterizada também como um movimento de contexto ideológico, no qual os termos gerenciamento participativo e democracia industrial foram evocados frequentemente como ideais. Assim, adquire contornos de um conceito globalizante no anseio de enfrentar questões ligadas à produtividade e à qualidade (PEREIRA; PEÇANHA, 2005).

Os anos 90 foram caracterizados, em termos da história da evolução da QVT, como seu momento de grande difusão em diversos países (Inglaterra, Hungria, Iugoslávia, Canadá, México, Brasil e Índia), passando assim a integrar o discurso acadêmico, a literatura científica sobre as organizações, os programas gerenciais de qualidade total, tendo também se popularizado (CAMPANA, 2011).

De acordo com Ts, Almeida e Borges (2007), atualmente, existem várias interpretações possíveis quanto à QVT, começando-se do foco clínico de ausência de doenças no âmbito pessoal, passando por melhorias ambientais no trabalho e atitudes proativas no sentido de melhorar as condições para o trabalhador. Outro foco são os recursos, objetos e procedimentos de ordem gerencial e estratégica empregados com o intuito de maximizar a satisfação dos servidores e majorar a produtividade organizacional. Além disso, percebe-se que têm sido incorporados novos conceitos e perspectivas aos estudos de QVT, ampliando a forma como o 
indivíduo, a organização e o trabalho são vistos sob esta corrente teórica (VELOSO; BOSQUETI; LIMONGI-FRANÇA, 2005).

Segundo Limongi-França (1996), Qualidade de Vida no Trabalho é o conjunto das ações de uma empresa que envolve a implantação das melhorias e inovações gerenciais e tecnológicas no ambiente de trabalho. A construção da Qualidade de Vida no Trabalho ocorre quando há integração entre a organização e as pessoas.

Para Rodrigues (1999), a QVT pode ser vista como uma abordagem sociotécnica em relação à organização do trabalho, tendo como base a satisfação do trabalhador no trabalho e em relação a ele. Neste contexto, a Qualidade de Vida no Trabalho, diretamente relacionada à satisfação e ao bem-estar do indivíduo na execução de suas tarefas, é indispensável à produtividade e à competitividade, sem as quais uma organização não sobrevive ao mercado.

Ferreira, Alves e Tostes (2009) analisam que a prática de QVT deve se apoiar em um modelo antropocêntrico de gestão do trabalho que, entre outras tarefas, preconize: valorizar os trabalhadores como principais protagonistas dos resultados, promover o reconhecimento individual e coletivo, estimular a criatividade e a autonomia na execução de tarefas, proporcionar o desenvolvimento pessoal e coletivo com base na cooperação intra- e intergrupal e possibilitar a participação efetiva, principalmente, nas decisões que afetem o bem-estar individual e coletivo.

A expressão QVT também pode ser associada ao sentimento que os trabalhadores desenvolvem em relação ao ambiente de trabalho e em relação à sua ocupação; pode também se referir às condições físicas presentes no ambiente de trabalho buscando a segurança do trabalhador tanto em relação a doenças ocupacionais como a acidentes de trabalho. Muitas vezes a QVT corresponde a programas implementados no ambiente organizacional, buscando aumentar a satisfação do trabalhador e sua motivação e com isso conseguir um aumento da produtividade (NESPECA; CYRILLO, 2011).

Segundo Ferreira, Alves e Tostes (2009) a implantação de um programa de QVT não deve ser uma medida tópica, isolada e circunscrita a uma fração do organograma da organização. Ela deve ser transversal a toda estrutura organizacional e conduzida com base em uma sinergia organizacional que implique efetivamente no compromisso de dirigentes e gestores, cooperação interdisciplinar de todos especialistas envolvidos e, sobretudo, parcerias intersetoriais e sindicais. 
A Qualidade de Vida no Trabalho relaciona-se também com a mobilização, o comprometimento pessoal, a participação com o bem-estar do funcionário na execução da tarefa na empresa, visando à consecução das metas da Qualidade Total. Um ambiente organizacional onde há uma gestão dinâmica e contingencial de fatores físicos, sociológicos, psicológicos e tecnológicos da organização do próprio trabalho torna-se saudável e mais propício ao aumento de produtividade. Este ambiente reflete no comportamento do funcionário no atendimento aos clientes e no contato com fornecedores (FREITAS; SOUZA, 2009).

De acordo com Nespeca e Cyrillo (2011), a QVT tem sido objeto de preocupação entre profissionais de diferentes áreas e muitas vezes é avaliada como sendo o bem-estar do trabalhador durante a execução de sua tarefa, por isso a satisfação no trabalho tem se tornado um conceito chave dentro das pesquisas de QVT.

Finalmente, observa-se que a QVT é um construto com característica multidimensional, individual, subjetivo, multidisciplinar e intersetorial. Desta forma, evidencia-se o quanto este construto é complexo tornando difícil sua conceituação (BARROS, 2017; GORDIA et al.,2011). Apesar disto, as várias definições encontradas sobre Qualidade de Vida no Trabalho revelam que existem alguns aspectos que são referidos com mais frequência.

Nas definições analisadas verifica-se que os estudos sobre QVT caminham em duas principais direções. Na primeira, no que tange à satisfação do trabalhador, as concepções buscam compreender o indivíduo como ser biopsicossocial. Ou seja, compreender o trabalhador no âmbito das questões biológicas (saúde física), psicológicas, sociais e ambientais (BARCAUI; LIMONGI-FRANÇA, 2014; GORDIA et al.,2011).

Na segunda direção, os autores observam que as preocupações sobre Qualidade de Vida no Trabalho têm extrapolado uma preocupação puramente legalista em relação ao trabalhador e adentrado também na esfera estratégica, delineando a QVT como parte da estratégia geral das organizações. Assim, são estabelecidos programas de QVT que visem proporcionar melhorias no âmbito laboral e ao mesmo tempo possibilitar maior satisfação aos servidores, fazendo com que haja um compromisso efetivo com os objetivos da organização e consequentemente a melhora dos resultados organizacionais (BARROS, 2017; VASCONCELOS, 2001). 


\section{Considerações finais}

Nos conceitos analisados no capítulo, percebe-se que vários fatores compreendem o que podemos denominar de Qualidade de Vida e Qualidade de Vida no Trabalho, visto que estes são compostos por aspectos sociais, organizacionais, ambientais, políticos, psicológicos e ergonômicos, entre outros. Isto posto, nota-se também que o conceito de QV e QVT é amplo, complexo, subjetivo e multidisciplinar, desta forma, relaciona-se com vários campos de estudo e não se isola em uma única definição.

Enfim, se no passado as empresas estavam interessadas apenas nas horas trabalhas e na quantidade de produtos produzidos, hoje, no atual cenário de competividade global é primordial manter os servidores motivados e satisfeitos para buscar o sucesso organizacional. Assim, é evidente que os aspectos que se relacionam com a Qualidade de Vida no Trabalho estão diretamente ligados aos índices de desempenho e produtividade. Portanto, são necessárias mais pesquisas e investimentos nessa área, devido às lacunas existentes na literatura e por ser um campo de estudo recente e ao mesmo tempo complexo.

\section{Referências}

BARCAUI, André; LIMONGI-FRANÇA, Ana C. Estresse, Enfrentamento e Qualidade de Vida: Um Estudo Sobre Gerentes Brasileiros. Rio de Janeiro: RAC- Revista de Administração Contemporânea, v. 18, n. 5, p. 670-694. 2014.

BARROS, Marizeth A. Qualidade de vida no trabalho (QVT): a percepção de docentes de uma instituição de ensino superior privada. Revista Espaço Acadêmico, v.16, n.188, p.38-46. 2017.

CAMPANA, Daniel P. Desenvolvimento e Avaliação de Projeto em Qualidade de Vida no Trabalho (QVT) - indicadores de produtividade e saúde nas organizações. 2011. Dissertação (Mestrado em Engenharia de Produção), EESC/USP, São Carlos.

CAMPANA, Daniel P.; PEÇANHA, Doris L. Avaliação quali-quantitativa de intervenção com Yoga na promoção da qualidade de vida em uma universidade. São Paulo: Bol. Acad. Paulista de Psicologia, v.78, n.1, p.199-218. 2010.

CHIAVENATO, Idalberto. Introdução à Teoria Geral da Administração. 7 ed. revisada e atualizada. Rio de Janeiro: Editora Campus - Elsevier, 2004. 
FERNANDES, Eda C. Qualidade de Vida no Trabalho: como medir para melhorar. Salvador: Casa da Qualidade Editora Ltda, 1996.

FERREIRA, Mário C.; ALVES, Luciana; TOSTES, Natalia. Gestão de Qualidade de Vida no Trabalho (QVT) no Serviço Público Federal: O Descompasso entre Problemas e Práticas Gerenciais. Brasília: Psicologia: Teoria e Pesquisa, v.25, n.3, p.319-327. 2009.

FREITAS, André L. P; SOUZA, Rennata G. B de. Um modelo para avaliação da Qualidade de Vida no Trabalho em universidades públicas. Niterói: Revista Eletrônica Sistemas \& Gestão, v.4, n.2, p.136-154. 2009.

GIMENES, Gabriel de F. Usos e significados da qualidade de vida nos discursos contemporâneos de saúde. Rio de Janeiro: Trabalho, Educação e Saúde, v.11, n.2, p. 291318. 2013.

GOMES, Igor G.; MENEZES, Ana C. P. Clima organizacional: uma revisão histórica do construto. Belo Horizonte: Psicologia em Revista, v. 16, n. 1, p. 158-179. 2010.

GOMES, Tais B. et al. Avaliação da qualidade de vida pós-implante de marcapasso cardíaco artificial. Goiânia: Revista Eletrônica de Enfermagem, v.13, n.4, 2011.

GORDIA, Alex P. et al. Qualidade de vida: contexto histórico, definição, avaliação e fatores associados. Ponta Grossa: Revista Brasileira de Qualidade de Vida, v.3, n.1, p. 40-52. 2011.

LANDEIRO, Graziela M. B. et al. Revisão sistemática dos estudos sobre qualidade de vida indexados na base de dados Scielo. Rio de Janeiro: Revista Ciência \& Saúde Coletiva, v.16, n.10, 2011.

LIMONGI-FRANÇA, Ana C. Indicadores empresariais de qualidade de vida no trabalho: esforço empresarial e satisfação dos empregados no ambiente de manufatura com certificação ISO 9000. 1996. Tese (Doutorado em Administração), FEA-USP, São Paulo.

LIPP, Marilda E. N.; ROCHA, J. C. Stress, hipertensão e qualidade de vida. Campinas: Papirus, 1996.

MACHADO, Eduarda L. et al. Qualidade de vida dos docentes: um estudo de caso. Três Corações: Revista da Universidade Vale do Rio Verde, v.9, n.2, p. 255-263. 2011.

MARTIN, A. J.; STOCKLER, M. Quality of life assessment in health care research and practice. Evolution and Health Professions, v.21, n.2, p. 141-156, 1998.

MAXIMIANO, Antonio C. A. Teoria geral da administração: da revolução urbana à revolução digital. $5^{\text {a }}$ ed. São Paulo: Atlas, 2005. 503p.

NESPECA, Milena; CYRILLO, Denise C. Qualidade de vida no trabalho de funcionários públicos: papel da nutrição e da qualidade de vida. Maringá: Acta Scientiarum. Health Science, v.33, n.2, p.187-195. 2011. 
PEREIRA, Valéria M.; PEÇANHA, Dóris L. Qualidade de vida no trabalho: que qualidade? In: XII SIMPEP- Relações de trabalho no contexto da Engenharia de Produção. Bauru: Universidade Estadual Paulista, 2005.

RODRIGUES, Marcus V. C. Qualidade de Vida no Trabalho: evolução e análise no nível gerencial. Petrópolis: Editora Vozes, 1999. 206p.

SEIDL, Eliane M. F.; ZANNON, Célia M. L. C. Qualidade de vida e saúde: aspectos conceituais e metodológicos. Rio de Janeiro: Cadernos de Saúde Pública, v.20, n.2, p. 580588. 2004.

SILVEIRA, Cristina D.; STIPP, Marluci A. C; MATTOS, Valéria Z. de. Fatores intervenientes na satisfação para trabalhar na enfermagem de um hospital no Rio de Janeiro. Goiânia: Revista Eletrônica de Enfermagem, v.16, n., p.100-108. 2014.

SOARES, Ana H. R. et al. Qualidade de vida de crianças e adolescentes. Rio de Janeiro: Ciência \& Saúde Coletiva, v.16, n.7. 2011.

TS, Wang Y.; ALMEIDA, Avelina F.; BORGES, Luiz C. O. Análise dos Efeitos da Terapia Comunitária Existente no Tribunal de Contas da União na Redução dos Níveis de Estresse dos Participantes. 2007. Monografia (Especialização em Gestão Estratégica de Pessoas no TCU), FIA/TCU, Brasília.

VASCONCELOS, Anselmo F. Qualidade de Vida no Trabalho: origem, evolução e perspectivas. São Paulo: Caderno de Pesquisas em Administração, v. 8, n. 1, p.23-35. 2001.

VELOSO, H. M; BOSQUETTI, A; LIMONGI-FRANÇA; A. C. A concepção gerencial dos programas de qualidade de vida no trabalho (QVT) no setor elétrico brasileiro. In: VIII SEMEAD - Seminários em Administração. São Paulo, 2005.

VIEIRA, A. A qualidade de vida no trabalho e o controle da qualidade total. Florianópolis: Editora Insular, 1996.

Como citar este artigo (Formato ABNT):

CAVALCANTE, Roberto M. da F.; PONTES, Karen, V.; BANDEIRA, Anselmo A.; ROCHA, André L. Qualidade de Vida e Qualidade de Vida no Trabalho: uma revisão bibliográfica. Id on Line Revista Multidisciplinar e de Psicologia, 2018, vol.12, n.40, p.21-31. ISSN: 1981-1179.

Recebido: 15.10 .2017

Aceito: 08.02.2018 